Although patients with preserved ejection fractions were largely excluded from percutaneous FMR trials, the findings mirror the MitraClip trial data. ${ }^{2}$ The Cardiovascular Outcomes Assessment of the MitraClip Percutaneous Therapy for Heart Failure Patients with Functional Mitral Regurgitation trial (COAPT) found a survival benefit but the Multicentre Study of Percutaneous Mitral Valve Repair MitraClip Device in Patients with Severe Secondary Mitral Regurgitation (MITRA-FR) did not. ${ }^{3,4}$ The patients in MITRA-FR had greater ventricular dysfunction and dilation, with resultant leaflet tethering. No strong conclusions can be made based on this data, but it would suggest this area is critical to future investigation. FMR pathoanatomic classification will help guide the utility of percutaneous interventions, but future research needs to evaluate the relative benefits compared with surgical intervention. These patients have poor long-term outcomes, making research into optimal care all the more important.

A critical area needing improvement in retrospective studies of FMR is the lack of serial echocardiographic follow-up. In this analysis, only $44 \%$ of patients had either echocardiographic or cardiology follow-up after
30 days. These studies often have few patients and low power, making surrogate endpoints, such as recurrent mitral regurgitation, helpful in determining the success of different interventions. Although not commonly performed, serial echocardiography data is best analyzed by longitudinal mixed-effects modeling. ${ }^{5}$ Incorporation of pathoanatomic features, improved follow-up, and comparison of surgical and percutaneous interventions are necessary to personalize interventions in a heterogenous group of patients with functional mitral regurgitation.

\section{References}

1. Hirji S, Cote C, Javadikasgari H, Malarczyk A, McGurk S, Kaneko T. Atrial functional versus ventricular functional mitral regurgitation: prognostic implications. $J$ Thorac Cardiovasc Surg. 2022;164:1808-15.e4.

2. Badhwar V, Alkhouli M, Mack MJ, Thourani VH, Ailawadi G. A pathoanatomic approach to secondary functional mitral regurgitation: evaluating the evidence. $J$ Thorac Cardiovasc Surg. 2019;158:76-81.

3. Stone GW, Lindenfeld J, Abraham WT, Kar S, Lim DS, Mishell JM, et al. Transcatheter mitral-valve repair in patients with heart failure. $N$ Engl J Med. 2018;379: 2307-18.

4. Obadia JF, Messika-Zeitoun D, Leurent G, Iung B, Bonnet G, Piriou N, et al. Percutaneous repair or medical treatment for secondary mitral regurgitation. $N$ Engl J Med. 2018;379:2297-306.

5. Laird NM, Ware JH. Random-effects models for longitudinal data. Biometrics. 1982;38:963-74.

\title{
Commentary: A riddle wrapped in a mystery inside an enigma
}

\author{
Jay K. Bhama, MD
}

Hirji and colleagues ${ }^{1}$ from Brigham and Women's Hospital report their nearly 20-year experience with mitral valve surgery in patients with atrial functional mitral regurgitation (A-FMR). The study details their experience with 94 patients who underwent mitral valve surgery in the setting of atrial fibrillation or left atrial dilatation

\footnotetext{
From the Division of Cardiovascular Surgery, University of Arkansas for Medical Sciences, and Department of Cardiothoracic Surgery, Baptist Heart Failure and Transplant Institute, Baptist Health Medical Center, Little Rock, Ark.

Disclosures: The author reported no conflicts of interest.

The Journal policy requires editors and reviewers to disclose conflicts of interest and to decline handling or reviewing manuscripts for which they may have a conflict of interest. The editors and reviewers of this article have no conflicts of interest.

Received for publication Jan 11, 2021; revisions received Jan 11, 2021; accepted for publication Jan 11, 2021; available ahead of print Jan 20, 2021.

Address for reprints: Jay K. Bhama, MD, Division of Cardiovascular Surgery, University of Arkansas for Medical Sciences, 4301 W Markham St, Little Rock, AR 72205-7199 (E-mail: jbhama@uams.edu).

J Thorac Cardiovasc Surg 2022;164:1818-9

0022-5223/\$36.00

Copyright (c) 2021 by The American Association for Thoracic Surgery

https://doi.org/10.1016/j.jtcvs.2021.01.041
}

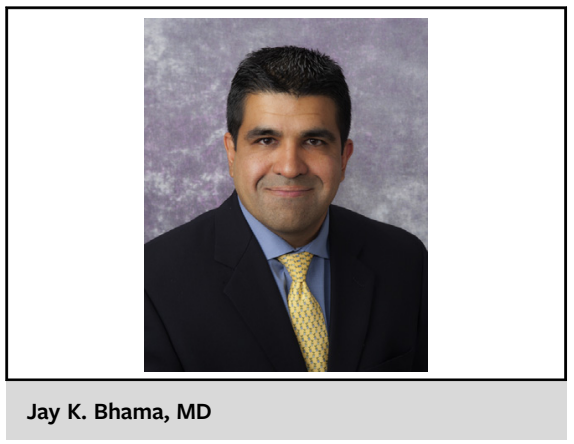

CENTRAL MESSAGE

Atrial functional mitral regurgi-

tation is an important prognostic

indicator in patients being

treated surgically for mitral

regurgitation. 
and preserved left ventricular function. In comparing this cohort of patients with a group of 84 patients who underwent mitral valve surgery for ventricular FMR, the authors found rather interesting and thought-provoking results.

First, and quite importantly, the authors identify important baseline cardiac morphological and functional differences between the 2 cohorts. Patients with A-FMR have more atrial fibrillation and right heart dysfunction and less left-sided heart failure with smaller left ventricle cavities and normal ejection fraction. Second, and also important, the authors demonstrate that patients with A-FMR have significantly better outcomes compared with their counterparts with ventricular FMR, which they identified as a significant predictor of midterm mortality in risk-adjusted analysis.

Given these differences, the authors deliver a message that is singular but not entirely simple. Early discrimination between these 2 etiologies of MR is important and may allow for better management of patients in the short and long-term. Unfortunately, as the authors adeptly allude to, A-FMR may be an elusive diagnosis often camouflaged by myriad other pathophysiologic issues that affect the cardio-respiratory system, including atrial fibrillation, heart failure with preserved ejection fraction, and potentially other coexisting cardiomyopathies that may complicate its diagnosis. Lack of concrete guidelines for the diagnosis of A-FMR further frustrate clinicians' ability to identify this pathology.
Other issues remain as well, including, as pointed out by the authors, the role of left atrial and annular dynamics in causing and perpetuating MR in these patients. This is especially important in patients with longstanding atrial fibrillation, where a strategy of rate control over rhythm control may expose the patient to higher left atrial pressures and lead to long-term remodeling of the atria with resultant valvular dysfunction. Whereas surgical therapies, as suggested by the authors, enjoy excellent short- and midterm outcomes, one must ask what role electrophysiology therapies and achievement of sinus rhythm should play in the therapeutic management of these patients.

Because there are many questions and not so many datadriven answers, Hirji and colleagues ${ }^{1}$ are to be congratulated on their efforts to elucidate the surgical outcomes in patients with A-FMR. Despite its observational, singlecenter, and nonrandomized nature, the data paint an important picture for surgeons and cardiologists alike depicting the importance of studying not only postoperative outcomes but also cardiac morphology and function at presentation. For most of us, A-FMR will continue to remain a riddle wrapped in a mystery inside an enigma until there is better understanding of how left atrial dysfunction influences cardiac-and especially mitral valvular-function.

\section{Reference}

1. Hirji AS, Cote CL, Javadikasgar HS, Malarczyk A, McGurk S, Kaneko T. Atrial functional versus ventricular functional mitral regurgitation: prognostic implications. J Thorac Cardiovasc Surg. 2022;164:1808-15.e4. 